\title{
EchoGéo
}

45 | 2018

Déclinaisons géographiques du changement social en Iran

\section{Le cinéma et la ville, un nouveau genre de documentaire en Iran}

Entretien avec Pouria Jahanshad, critique de cinéma documentaire, réalisé par Mina Saîdi-Sharouz en octobre 2018.

Pouria Jahanshad et Mina Saïdi-Sharouz

\section{(2) OpenEdition}

\section{Journals}

Édition électronique

URL : https://journals.openedition.org/echogeo/16051

DOI : $10.4000 /$ echogeo.16051

ISSN : 1963-1197

Éditeur

Pôle de recherche pour l'organisation et la diffusion de l'information géographique (CNRS UMR 8586)

Référence électronique

Pouria Jahanshad et Mina Saïdi-Sharouz, « Le cinéma et la ville, un nouveau genre de documentaire en Iran », EchoGéo [En ligne], 45 | 2018, mis en ligne le 05 novembre 2018, consulté le 10 août 2021. URL : http://journals.openedition.org/echogeo/16051; DOI : https://doi.org/10.4000/echogeo.16051

Ce document a été généré automatiquement le 10 août 2021.

EchoGéo est mis à disposition selon les termes de la licence Creative Commons Attribution - Pas d'Utilisation Commerciale - Pas de Modification 4.0 International (CC BY-NC-ND) 


\section{Le cinéma et la ville, un nouveau genre de documentaire en Iran}

Entretien avec Pouria Jahanshad, critique de cinéma documentaire, réalisé par Mina Saîdi-Sharouz en octobre 2018.

\section{Pouria Jahanshad et Mina Saïdi-Sharouz}

1 En Iran, le cinéma documentaire a connu de nombreuses périodes. Selon la situation politique, l'objet et le style ont beaucoup évolué. Après la révolution de 1979, beaucoup de films s'intéressaient au monde rural. Le régime islamique, en rupture avec le projet d'occidentalisation du Shah, tournait le dos à la ville et ne voyait pas d'un bon œil les films urbains. Montrer les folklores des villages permettait aux cinéastes de ne pas franchir ces lignes rouges.

2 Ce n'est que depuis une dizaine d'année, qu'à nouveau, les jeunes documentaristes s'intéressent aux grandes villes, suivant en cela l'évolution du cinéma de fiction. Ces jeunes réalisateurs s'inspirent notamment des cinéastes précurseurs des années 1960 à 1980 tels que Forough Farokhzad, Kamran Shirdel, Rakhshan Bani Etemad, pour porter un regard critique sur la ville et sur les ségrégations sociales.

$\mathrm{Au}$ lendemain de la révolution, le ministère de l'Urbanisme et du logement a commandé trois films autour de la situation de l'habitat à Téhéran, confiés à trois grands réalisateurs de documentaire. Les films produits sont Le locataire d'Ebrahim Mokhtari, Le foncier urbain de Fereydoun Javadi et enfin, La crise de logement et immigration de Mohamad Tahami Nejad. Malgré leur grande qualité artistique et documentaire, ces films seront très peu diffusés auprès du grand public. Cependant, le fait qu'ils aient été regardés et utilisés par les responsables du pays pour ajuster leurs politiques urbaines, mérite d'être souligné.

3 Aujourd'hui, les films « urbains » en Iran sont en pleine effervescence. La plupart des réalisateurs tels que Pirouz Kalantari, Hadi Afarideh et bien d'autres, dressent des portraits des citadins et des espaces urbains en mutation. Ils donnent à voir le singulier dans l'uniformisation d'une métropole en pleine transformation. D'autres réalisateurs s'intéressent aux sujets plus sociologiques et filment dans des milieux d'enfermement, au sens donné par Foucault (école, atelier, prison, etc.). Face aux nombreuses 
contraintes qu'ils doivent contourner dans ce type de situation - obtenir l'adhésion des personnes filmées et les autorisations des institutions, les cinéastes iraniens sont amenés à inventer de nouvelles formes cinématographiques. Parmi cette vague, nous pouvons citer deux films assez remarquables: d'une part, Les rêves sans étoiles de Mehrdad Oskouee, un documentaire sur des jeunes filles mineures dans le milieu carcéral ; d'autre part, Les accusés $d u 20^{\circ}$ district, film de Hesam Eslami où le réalisateur partage littéralement sa vie avec une bande de jeunes délinquants, construisant avec eux une amitié qui s'inscrit dans la durée, son film étant le fruit d'un contrat éthique entre lui et les personnages du film.

Ce type de films se détache aujourd'hui des très nombreuses productions qui, régulièrement, sont réalisés sans véritable problématique ni invention formelle. Pouria Jahanshad, critique de cinéma, partage avec nous ce questionnement quant à l'évolution de ces films.

4 - Mina Saidi (MS). Depuis une dizaine d'années, en Iran, on parle du documentaire "urbain ». Beaucoup de films sont réalisés sur le thème de la ville. Au festival du cinéma documentaire Haghighat, un prix est même consacré à cette catégorie de film. Pouvez-vous nous dire de quoi il s'agit vraiment?

5 - Pouria Jahanshad (PJ). Depuis 2005, un service au nom de "Tasvir-é-shahr», appartenant à la municipalité de Téhéran, finance des films qui donnent à voir les différents aspects de Téhéran, en rapport avec les préoccupations des autorités de la ville. Plus de cent films ont été réalisés, au départ sur les personnages, puis ensuite sur les aspects physiques comme les arbres, les avenues, le patrimoine, les places, etc. Mais dans l'ensemble, ces films restent assez descriptifs et sont peu diffusés en dehors du circuit de la Mairie.

6 Ces films, qu'on appelle en Iran "mostanad-é-shahri », se distinguent à mon avis du documentaire social. Ce sont des films axés sur les aspects techniques et physiques et ils ne placent pas l'humain au centre de leur intérêt. Nous avons une autre catégorie de films appelée documentaire social et qui s'applique plutôt aux pathologies sociales, sans traiter non plus les aspects politiques des problèmes sociaux. Dans l'ensemble, peu de films documentaires traitent les questions urbaines sous l'angle politique et social.

Depuis 2014, nous avons créé une association du nom de Rokhdad-é-tazeh, pour réfléchir entre autres sur les thèmes concernant la ville. Nous avons formé des groupes de réflexion autour des questions juridiques, l'empowerment et la participation des citoyens à la production urbaine. Nous travaillons également sur la question de l'informalité dans la ville. Nous avons décidé depuis trois ans d'organiser des séances mensuelles de projection-débat concernant les films documentaires qui interrogent le politique et cherchent à apporter des changements de regard sur les problèmes sociaux. Nous invitons à la fois les réalisateurs, les activistes, les sociologues et les professionnels de la ville à s'exprimer sur le sujet afin de théoriser ensemble ces débats.

Nous trouvons que la plupart des films produits et primés en Iran et même dans le monde, ont un regard neutre sur les situations. Par exemple, nous avons consacré trois séances aux films sur la vie informelle à Téhéran. Des activités qui sont interdites et qui ne bénéficient d'aucun droit juridique comme les spectacles de rue et qui questionnent les formes de la vie urbaine, en tension avec le discours dominant. Nous mettons le projecteur sur ceux dont l'existence aussi bien que les activités restent dans l'ombre. Les films projetés questionnent les pratiques informelles. Par exemple, Les rois de la rue de Zeynab Tabrizi et Parviz Khoshdel à propos du fondateur de parcours dans la cité 
d'Ekbatan et sa relation à la ville ; Sur le sous-sol de Mahan Khamamipour, à propos des danseurs de rue et enfin, Une valse pour Téhéran de Zeynab Tabrizi sur des jeunes musiciens de rue. Ces pratiques font elles parties des pratiques quotidiennes de la ville ou bien présentent-elles une forme de résistance à la vie formelle?

Nous avons également consacré plusieurs séances à la question du genre avec un angle nouveau. En général, en Iran, les films cherchent à "victimiser» les femmes en montrant leur côté fragile et sensible. Ils reflètent un regard masculin et dominant sur les rapports homme-femme dans l'espace. Nous essayons de briser ce regard en montrant la résistance et l'insoumission des femmes dans la vie quotidienne. Nous nous référons à des films cultes comme par exemple News from home de Chantal Akermann, qui montre la ville comme l'espace du quotidien et d'une résistance à toute politique imposée par le haut. Nous sommes également témoins d'une récupération du foncier du centre-ville par les municipalités, qui aboutit à la marginalisation d'une certaine population et son expulsion vers la périphérie.

9 Un autre thème abordé lors de nos projections-débats est la question des migrants en ville. Plusieurs réalisateurs iraniens ont filmé les migrants comme des victimes, pour susciter chez le spectateur un sentiment de pitié et de culpabilité. Nous critiquons ce type de films en privilégiant ceux qui nous renvoient à notre propre regard sur les migrants, des films qui suggèrent que chacun de nous pourrait être un migrant potentiel. D'ailleurs, nous mettons en parallèle les films sur la diaspora iranienne à l'étranger, avec parfois les mêmes difficultés que les afghans en Iran.

10 - MS. Quelles sont les prochaines thématiques de votre programme?

11 - PJ. Nous allons nous concentrer sur la question de l'architecture et des mécanismes du contrôle dans la ville. Nous observons que le contrôle ne s'effectue pas uniquement par le biais de la répression physique, mais aussi par des moyens plus sournois qui nécessitent la collaboration des intermédiaires tels que les professionnels. Ces intermédiaires qui peuvent être des architectes ou des urbanistes, participent à la légitimation des décisions prises par le haut en prêtant leurs noms et leur réputation. Les grands architectes cautionnent ainsi des bâtiments construits sur du foncier public en faveur des privés. Nous cherchons à analyser ce qui se cache derrière ces innovations artistiques et techniques.

12 Nous valorisons dans nos discussions la posture de l'architecte engagé et militant, et une architecture pouvant contribuer à améliorer la situation d'un pays et d'une société. Nous essayons de montrer que chaque problème urbain a trois faces. Les projets concernent les autorités, les professionnels et les habitants. Tandis que nous pointons l'État comme responsable de la situation, les professionnels continuent à légitimer l'action publique. Le regard des architectes et des urbanistes en Iran est un regard du haut vers le bas. C'est le moment de l'autocritique.

13 Le documentaire Lettre au Ministre, réalisé par Mina Saïdi-Shahrouz sur le quartier Sirous, est un exemple de film mettant en scène les rapports de force entre les différents acteurs. Il interroge la responsabilité des professionnels dans la prise en compte des intérêts collectifs et critique les décisions prises par le haut sans se concerter avec les habitants. Ce type de film constitue des archives de la ville très précieuses.

14 - MS. Quelles sont les nouvelles tendances de «films urbains » à votre avis? 
15 - PJ. Personnellement, j'étais très optimiste pour le cinéma documentaire en Iran, mais je me pose des questions sur son évolution actuelle. Il est de plus en plus formaté. Nous avons des politiques de financement et des critères de vente qui contribuent à neutraliser les films documentaires en Iran. La tendance actuelle est d'opter pour une dramaturgie qui puisse séduire le public. Les documentaires s'éloignent du réel et nous mentent. Ce phénomène est mondial et en Iran ces films perdent leur impact sur le politique. La recherche de la forme et de l'esthétique devient le premier souci des critiques du cinéma en Iran. Le souci de plaire à un plus grand nombre pousse les réalisateurs à utiliser la forme fictionnelle qui envoute et fascine mais ne fait pas avancer la société.

16 À Rokhdadé tazeh, nous privilégions un cinéma plus direct et plus franc. Nous ne cherchons pas à faire pleurer le public sur le sort des réfugiés afghans, mais plutôt à en questionner les raisons. Pour nous, il ne suffit pas de montrer ou donner la parole aux exclus de la société, ce qui compte est la manière dont on les montre.

17 - MS. En 2017 nous avons organisé avec le comité ethnographique de Jean Rouch une projection au Musée de l'Homme à Paris sur le documentaire iranien et nous avons choisi le film Les rêves sans étoiles de Mehrdad Oskouee et Les accusés du $20^{e}$ district de Hesam Eslami. À mon avis ces films qui donnent la parole aux filles incarcérées et à des jeunes délinquants de Téhéran représentent toute une nouvelle approche. Le documentariste est plus impliqué dans la relation avec ceux qu'il filme. Qu'en pensezvous?

18 - PJ. Oui, ce sont de bons exemples. Mais personnellement je préfère le film de Hesam Eslami, car celui de Mehrdad Oskouee est compassionnel mais ne nous permet pas de nous remettre en question. Il choisit l'angle des droits de l'Homme et de la pitié pour ces filles victimes de leurs parents. Cela pourrait au mieux aboutir aux actions de charité. En revanche, Hesam Eslami montre des jeunes en marge mais qui ne suscitent pas de pitié. Il nous aide à être de leur côté et à penser qu'ils sont des êtres comme nous. Il atténue les frontières entre les gens «normaux » et les autres. Ce ne sont pas des raisons personnelles qui les ont entrainés sur ce chemin, mais les problèmes sociaux. Les différentes formes de vie produisent des comportements différents. Nous pouvons nous retrouver à travers leurs vies. Un système totalitaire accuse les personnes qui sont en difficulté d'avoir été coupables de leur situation.

19 Les films qui réussissent du point de vue commercial sont des films qui suivent les schémas hollywoodiens selon lesquels il existe d'une part des pauvres et des victimes, et, de l'autre, des héros qui vont sauver ces êtres perdus. Mais dans ce film, nous ne pleurons pas et nous n'avons pas non plus envie de sauver le personnage principal car il va s'en sortir tout seul. Le réalisateur a été là comme un ami et non comme un sauveur. Ils ne sont pas si différents au fond. Est-ce que la situation à l'extérieur est meilleure? De manière générale, la solution est politique et la charité ne peut rien. Il faut changer notre regard pour pouvoir changer la situation. L'art réside dans la vie quotidienne. 


\section{AUTEURS}

\section{POURIA JAHANSHAD}

Pourra Jahanshad est un critique du cinéma documentaire iranien. Il est diplômé du Tehran Institute of advanced directing et il est co-directeur depuis 2015 de Rokhdad-é-tazeh Mostanad, un centre de recherche et de critique sur le cinéma documentaire en Iran. Il a récemment publié : - Jahanshad P., 2017. De la philosophie à la politique. Critique du cinéma documentaire. In Centre du cinéma documentaire et expérimental d'Iran, Reality and Imagination in Documentary Cinema. Téhéran, Yazda publications.

- Jahanshad P., 2017. In Yaghoubi M. (ed.), Winter of 66. Teheran, Afaraz publication.

\section{MINA SAÏDI-SHAROUZ}

Mina Saïdi-Sharouz, minasaidi@yahoo.com, est architecte et géographe. Enseignante à l'École Nationale Supérieure d'Architecture de Paris-La Villette (ENSAPLV), elle est membre du Laboratoire Architecture Anthropologie (LAVUE/CNRS). Elle a publié récemment :

- Saïdi-Sharouz M., 2018. L'intégration des migrants dans le centre de Téhéran. La communauté des Ramshehi à Cyrus. In Raulin A., Parsapajouh S. et Blanc-Chaléard M.C. (dir.), Ces villes-là. Actualité de Colette Pétonnet. Paris, Presses Universitaires de Paris Nanterre, p.135-158.

- Saïdi-Sharouz M., 2015. « Les touristes iraniens à Istanbul, les fourmis voyageuses de la mondialisation ». Hommes \& Migrations, 2015/4, n 1312, p. 81-89.

- Saïdi-Sharouz M. (dir.), 2013. Le Téhéran des quartiers populaires. Transformation urbaine et société civile en République Islamique. Paris, Karthala-IFRI, 265 p. 